\title{
Operational status of TAMA300 with the seismic attenuation system (SAS)
}

\author{
R Takahashi ${ }^{1}$, K Arai $^{1}$, D Tatsumi ${ }^{1}$, M Fukushima ${ }^{1}$, T Yamazaki $^{1}$, \\ M-K Fujimoto ${ }^{1}$, K Agatsuma $^{2}$, Y Arase $^{3}$, N Nakagawa ${ }^{2}$, A Takamori ${ }^{4}$, \\ K Tsubono ${ }^{5}$, R DeSalvo ${ }^{6}$, A Bertolini ${ }^{7}, \mathrm{~S} \mathrm{Márka}^{8}$ and V Sannibale ${ }^{6}$ \\ (the TAMA Collaboration) \\ ${ }^{1}$ National Astronomical Observatory of Japan, Mitaka, Tokyo 181-8588, Japan \\ 2 Institute for Cosmic Ray Research, University of Tokyo, Kashiwa, Chiba 277-8582, Japan \\ ${ }^{3}$ Department of Astronomy, University of Tokyo, Bunkyo, Tokyo 113-0033, Japan \\ ${ }^{4}$ Earthquake Research Institute, University of Tokyo, Bunkyo, Tokyo 113-0032, Japan \\ ${ }^{5}$ Department of Physics, University of Tokyo, Bunkyo, Tokyo 113-0033, Japan \\ ${ }^{6}$ California Institute of Technology, Pasadena, CA 91125, USA \\ ${ }^{7}$ Deutsches Elektronen-Synchrotron, Hamburg 22607, Germany \\ ${ }^{8}$ Columbia University in the City of New York, New York, NY 10027, USA \\ E-mail: ryu.takahashi@nao.ac.jp
}

Received 5 November 2007, in final form 25 December 2007

Published 15 May 2008

Online at stacks.iop.org/CQG/25/114036

\begin{abstract}
TAMA300 has been upgraded to improve the sensitivity at low frequencies after the last observation run in 2004. To avoid the noise caused by seismic activities, we installed a new seismic isolation system-the TAMA seismic attenuation system (SAS). Four SAS towers for the test-mass mirrors were sequentially installed from 2005 to 2006. The recycled Fabry-Perot Michelson interferometer was successfully locked with the SAS. We confirmed the reduction of both length and angular fluctuations at frequencies higher than $1 \mathrm{~Hz}$ owing to the SAS.
\end{abstract}

PACS number: $04.80 . \mathrm{Nn}$

(Some figures in this article are in colour only in the electronic version)

\section{Overview}

The laser interferometer gravitational wave detector TAMA300 [1] has been operating since 1999. TAMA300 is located at the Mitaka campus of the National Astronomical Observatory of Japan. The aims of this project are to develop advanced techniques for future kilometric ground-based interferometers like LCGT [2] and to detect gravitational waves that may occur within our local group of galaxies. Not only TAMA300 but also several other interferometric 
detectors (GEO [3], LIGO [4] and VIRGO [5]) are presently in operation to detect the first gravitational wave signals.

TAMA300 performed nine observation runs up until 2004. A total of $3000 \mathrm{~h}$ data was accumulated [6]. After the last observation run, TAMA300 was upgraded to reduce the lowfrequency noise. The alignment noise was dominant at frequencies lower than $200 \mathrm{~Hz}$ [7]. Reduction of the angular fluctuation of mirrors by a powerful seismic isolation system was required to make the narrow band alignment control possible.

Development of the TAMA SAS [8] started in 1999 in cooperation with the LIGO SAS group to improve the vibration isolation system in TAMA300. The experiments using the TAMA SAS prototype [9] were finished in 2002. We started manufacturing and assembling the SAS for TAMA300 in 2003 . The plan is to upgrade the isolation system for the four test-mass mirrors. In the former isolation system, the mirror was suspended by a doublependulum which was placed on an optical table supported by a three-layer stack [10] and an active isolation system with pneumatic actuators [11]. The new isolation system consists of a horizontal 5-layer pendulum and a 3-layer vertical isolation.

The first SAS was installed in the west-end chamber (EM1) in 2005. The second SAS was installed in the east-west front chamber (NM1) in 2006, thus forming a $300 \mathrm{~m}$ cavity with the first SAS. Then, the SAS pair was installed in the north-south arm (NM2, EM2) by the end of 2006. We could successfully lock the recycled Fabry-Perot Michelson interferometer with all the four SASs in June 2007. The improvement of the interferometer sensitivity by the SAS was confirmed, as described in this paper.

\section{Seismic attenuation system (SAS)}

\subsection{Structure}

The fundamental structure of SAS is similar to the super attenuator [12] of VIRGO, while the SAS utilizes a special spring geometry to replace the magnetic anti-springs. Figure 1 shows a schematic view and a picture of the TAMA SAS. An inverted pendulum (IP) [13] was employed for the horizontal isolation at low frequencies. The two spring constants due to the combination of gravity anti-spring and the elastic spring of the flexible joint were tuned to realize a natural resonant frequency lower than $100 \mathrm{mHz}$. Two stages of vertical filters using monolithic geometrical anti-spring (MGAS) [14] are suspended from the IP. Cantilever springs with anti-spring force generated by radial horizontal compression can realize vertical resonance lower than $0.5 \mathrm{~Hz}$. The flexible joints for the IP and the MGASs are made of maraging steel which has a very large tensile strength of more than $2 \mathrm{GPa}$. A payload, which consists of a platform, an intermediate stage and a test-mass with a recoil-mass [15], is suspended by the vertical filter chain. The platform is aligned by eight magnet-coil actuators. Four mini-MGAS filters are embedded in the platform for vertical isolation. The test-mass suspension is compatible with the former double-pendulum. Eddy current damping is applied on the intermediate stage. The test-mass is actuated from the coaxial recoil-mass through four additional magnet-coil actuators.

\subsection{Control system}

The control system for the TAMA SAS is handled by a digital system using a commercial PXI-bus target produced by National Instruments (NI). An embedded controller has a CPU (typically Intel Pentium III/1.26 GHz) operated by a real-time operating system. Analog signals are processed through ADC (8 channels, 16-bit, $\left.333 \mathrm{kS} \mathrm{s}^{-1}\right)$ and DAC (8 channels, 


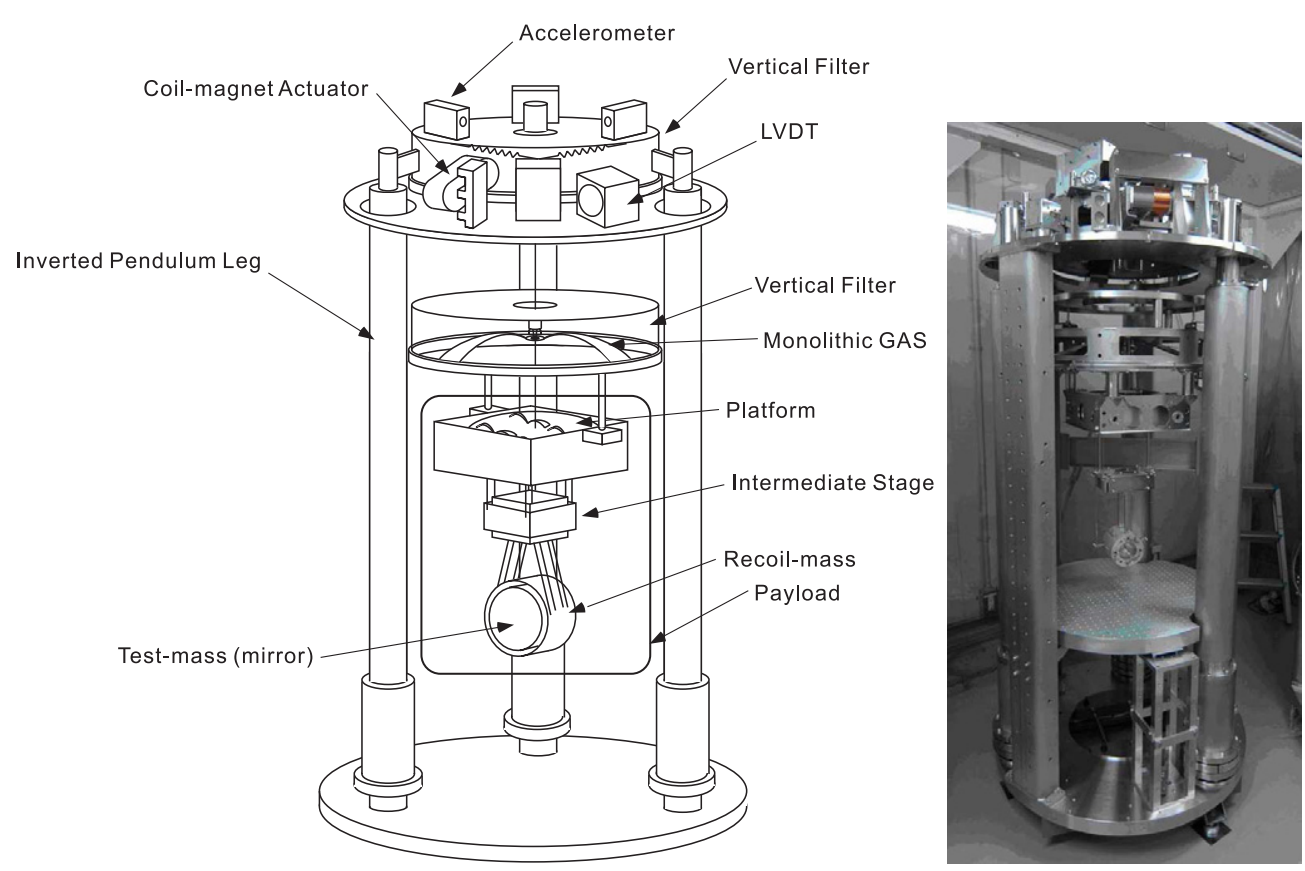

Figure 1. Schematic view and picture of the TAMA SAS.
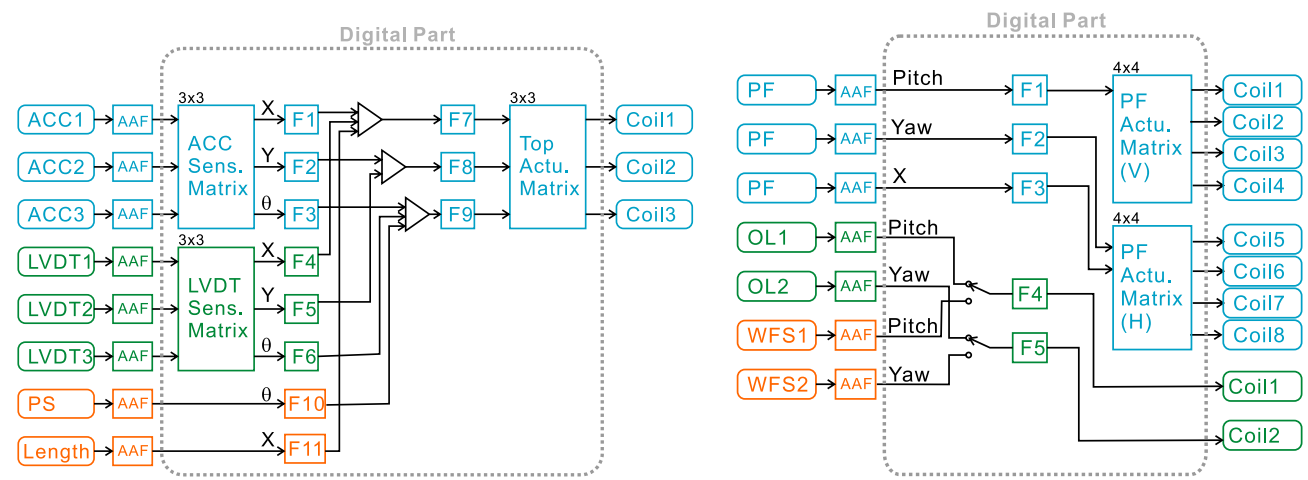

Figure 2. Schematic diagram of the control system for the TAMA SAS. There are two kinds of control systems - the IP control (left) and the PF-TM control (right). The digital parts synthesize servo filters.

16-bit, $1 \mathrm{MS} \mathrm{s}^{-1}$ ) modules. Programs are coded on a host PC using NI LabVIEW. The programs are compiled on the host PC, uploaded into the remote target and executed there.

We implemented two kinds of control systems. One is the IP control shown schematically on the left side of figure 2. There are three accelerometers (ACC) [16], three linear variable differential transformers (LVDT) [17] as position sensor and three coil-magnet actuators [18] on the top of the SAS to control the horizontal directions. A LVDT and a coil-magnet actuator are employed also for the vertical direction. Since the accelerometers, the LVDTs and the actuators are not aligned to the direction of IP's eigenmodes $(X, Y$ and $\theta)$, the sensing and 
Table 1. Fundamental frequencies of the inverted pendulum.

\begin{tabular}{lcll}
\hline & $X[\mathrm{mHz}]$ & $Y[\mathrm{mHz}]$ & $\theta[\mathrm{Hz}]$ \\
\hline NM1 & 50 & 60 & 0.50 \\
EM1 & 150 & 50 & 0.54 \\
NM2 & 30 & 60 & 0.52 \\
EM2 & 50 & 70 & 0.50 \\
\hline
\end{tabular}

driving signals must be diagonalized to the directions of $X, Y$ and $\theta$. Six channels are assigned to the ADC inputs of the digital controller for the accelerometers and the position sensors. Analog anti-aliasing filters (AAF) are mounted in front of the ADC inputs. Three channels are assigned to the DAC outputs for the actuators. The main process of the digital controller is the diagonalization and filtering of the input signals, as well as generating control voltages on the three actuators corresponding to three virtual actuators acting on each of the eigenmodes of the IP. First, the diagonalization matrix was decided using natural modes which appear in the measured transfer functions from each actuator to each sensor. Second, optimal servo filters were designed using the measured transfer functions from a diagonalized virtual actuator to a virtual sensor. The loop rate of the process is $250 \mathrm{~Hz}$. The unity gain frequency of the open loop is at $2 \mathrm{~Hz}$. Additionally, there are a global control loop for the cavity length and a damping control loop for excited torsion modes using a differential photo sensor (PS) and the virtual actuator acting on $\theta$. These torsion modes are due to the moment of inertia of the payload suspended by a single wire from the IP. The fundamental mode has a resonant frequency of $\sim 40 \mathrm{mHz}$ and a long settle time of $\sim 1000 \mathrm{~s}$.

The other is the platform and test-mass (PF-TM) control shown schematically on the right side of figure 2. Signals of the test-mass rotation (pitch and yaw) detected by a local optical lever (OL) are fed back to the test-mass actuators. The loop rate of this process is $1 \mathrm{kHz}$. The unity gain frequency of the open loop is at 2-3 Hz. After the cavity is locked, the sensing signals are replaced by global signals from the interferometer acquired by the wave front sensing (WFS) method [19, 20]. We could successfully change the control signals from the optical lever to the WFS without the loss of lock. Additionally, there are control loops for the platform. At present, this function is used only to apply DC offsets for the initial alignment of the test-mass.

\subsection{Characteristics of the inverted pendulum}

The fundamental frequencies of the inverted pendulum (IP), which characterize the quality of each SAS, are shown in table 1 . Most of the horizontal modes ( $X$ and $Y$ ) were tuned to be lower than $100 \mathrm{mHz}$ except for EM1 in which an asymmetry of the elastic spring components of the IP caused the large splitting of the modes. The frequencies of rotational mode $(\theta)$ are $\sim 0.5 \mathrm{~Hz}$. The natural quality factor $Q$ of the horizontal modes was very small ( 1 or less). This property causes the $1 / f$ (where $f$ is the frequency) behavior of the vibration isolation function. As a result, the attenuation from the ground motion of the IP was about $-25 \mathrm{~dB}$ at $1 \mathrm{~Hz}$. A prototype TAMA SAS had $Q$ of $\sim 10$ at $50 \mathrm{mHz}$ [9]. It is likely that vacuum-compatible stiff wires used for electric signals added mechanical loss. 


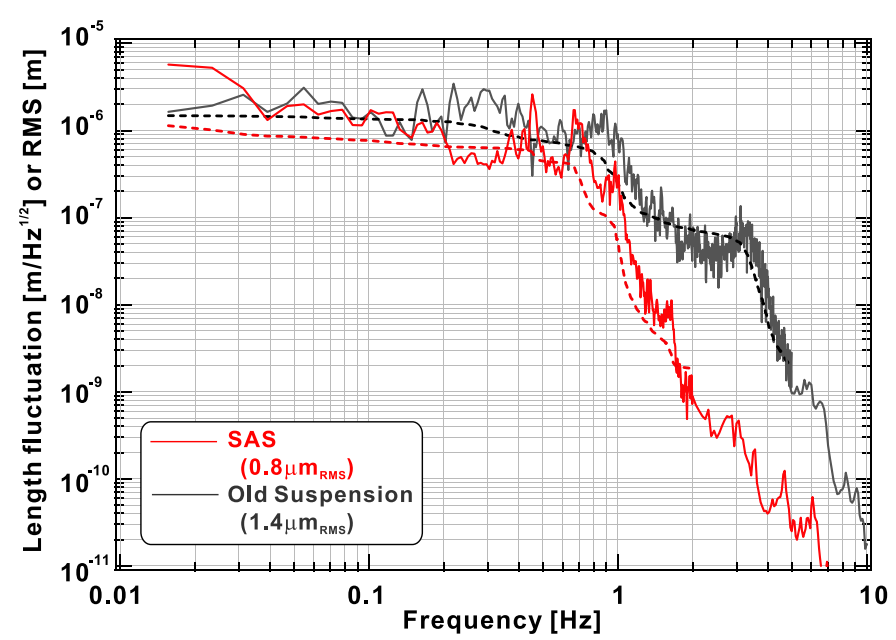

Figure 3. Length fluctuation of the $300 \mathrm{~m}$ cavity measured by the feedback signals to lock the cavity. The spectrum density and RMS integrated from $50 \mathrm{mHz}$ are indicated by solid line and dash line, respectively.

\section{Confirmation of performance}

\subsection{Configurations of the interferometer}

We installed the SAS one by one and confirmed its performance using the TAMA interferometer step-by-step: Step-1, one arm; Step-2, locked Fabry-Perot (FP); Step-3, recycled Fabry-Perot Michelson interferometer (FPMI).

In Step-1, one arm with the SAS was compared with the other arm with the old suspension. The length fluctuation of the arm cavity was measured by the feedback signal fed to the laser in order to lock the cavity.

In Step-2, each arm cavity with the SAS was locked differentially. We call this configuration the locked FP. The inline arm cavity was locked by actuating the laser frequency for stabilization of the light source, while the perpendicular arm cavity was locked to this stabilized light by actuating the front mirror of the perpendicular arm, so-called mass-lock. Since the power recycling mirror was misaligned, the light power was very small.

Step-3 is close to the full configuration with power recycling. The recycling mirror and Michelson part are locked using signals demodulated at the third-harmonic frequency [21]. Only the signal for the Michelson part is changed to signal demodulated at the first-harmonic frequency after the lock acquisition.

\subsection{Length performance}

The length fluctuation of the cavity was confirmed in the Step-1 configuration. The global control for the cavity length was not working. The arm with the SAS showed reduced fluctuation above $0.1 \mathrm{~Hz}$ as compared with the arm with the old suspension, as shown in figure 3. Attenuation better than $1 / f^{5}$ was achieved above $1 \mathrm{~Hz}$. The mechanical resonances of multi-pendulum around $1 \mathrm{~Hz}$ and micro-seismic motion around $0.3 \mathrm{~Hz}$ are successfully damped by the digital servo system. RMS fluctuation integrated from $50 \mathrm{mHz}$ was $0.8 \mu \mathrm{m}$ with the SAS. This is comparable with RMS of $1.4 \mu \mathrm{m}$ with the old suspension. 


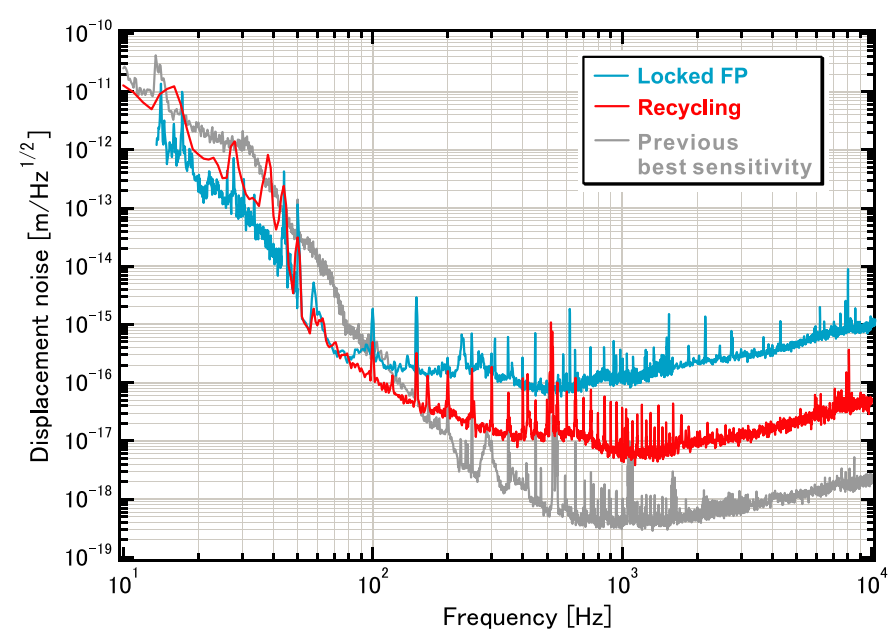

Figure 4. Sensitivity of TAMA 300 with the SAS in Step-2 configuration (locked FP) and Step3 configuration (recycling). The previous best sensitivity is also shown. Please note that the worsened sensitivity above $150 \mathrm{~Hz}$ is due to the incomplete commissioning of the interferometer. The SAS has no effect above $100 \mathrm{~Hz}$.

Improvement of the displacement noise at $0.1-100 \mathrm{~Hz}$ was confirmed in the Step-2 configuration as compared with the previous best sensitivity (figure 4). The sensitivity around $1 \mathrm{kHz}$ was far from the best one because of small light power.

In the Step-3 configuration, the sensitivity of around $1 \mathrm{kHz}$ was close to the best one as shown in figure 4. The improved frequency region was extended to $150 \mathrm{~Hz}$. Please note that the worsened sensitivity above $150 \mathrm{~Hz}$ is due to the incomplete commissioning of the interferometer. The light power at the dark port was not large enough to obtain the sensitivity consistent with the best one. The SAS has no effect above $100 \mathrm{~Hz}$.

An alignment noise was still dominant at $10-50 \mathrm{~Hz}$. One thing, however, is certain: the signals acquired by the WFS are larger than the signals acquired by the local optical lever. The WFS may couple to jitters of the incident beam to the arm cavity. Therefore, a common mode signal should be fed back not to the test-mass mirrors but to the incident beam. We need a new servo topology to solve this problem.

\subsection{Angular performance}

We confirmed the angular fluctuation of the test-mass using an optical lever. The fluctuation with the SAS showed improvement above $1 \mathrm{~Hz}$ as compared with the fluctuation with the old suspension as shown in figure 5. Sensing noise of the optical lever is lying around $10^{-9} \mathrm{rad} \mathrm{Hz}^{-1 / 2}$. Although the uncontrolled RMS fluctuation with the SAS (12 $\mu$ rad for yaw) was larger than the fluctuation with the old suspension $(1.0 \mu \mathrm{rad})$, we could set the bandwidth of the alignment control servo to $2-3 \mathrm{~Hz}$. It was $10-20 \mathrm{~Hz}$ previously. The many resonant peaks below $3 \mathrm{~Hz}$, due to the multi-pendulum, were damped using a servo filter with many pole-zero pairs. It is difficult to construct such a filter by an analog circuit at frequencies below $1 \mathrm{~Hz}$. The residual RMS of angular fluctuation was $0.2 \mu \mathrm{rad}$ with the control. This will result in a reduction of the noise due to the alignment control system which limited the former sensitivity of TAMA300. 

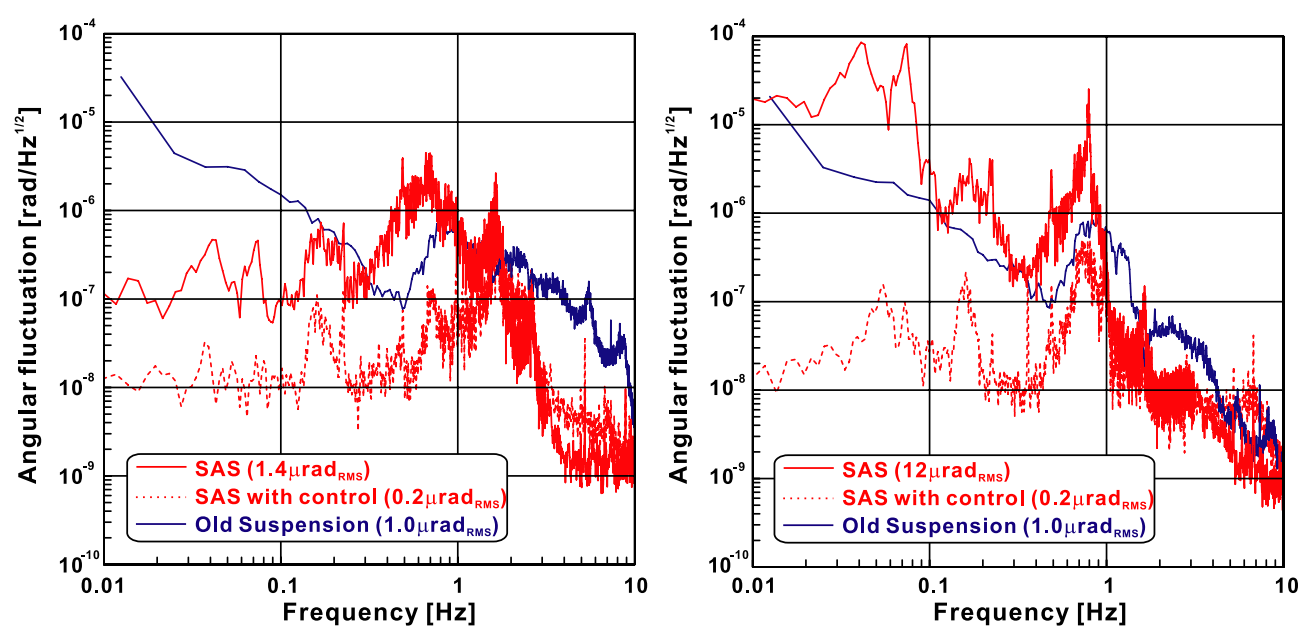

Figure 5. Angular fluctuation of the north-south front test-mass (NM2) measured by an optical lever. The spectrum density in the pitch direction (left) and in the yaw direction (right) are shown, respectively.

\section{Summary}

Installation of the SAS into TAMA300 was completed. The recycled FPMI was successfully locked using the established control system for the SAS. The length fluctuation of the $300 \mathrm{~m}$ cavity was improved above $0.1 \mathrm{~Hz}$ owing to the SAS. The improved angular fluctuation of the test-mass will result in a reduction of the alignment noise.

\section{Acknowledgments}

This work was supported by the Grant-in-Aid for Scientific Research of the Ministry of Education, Culture, Sports, Science and Technology (09NP0801 in Creative Scientific Research and 415 in Priority Areas). Developments of the TAMA SAS were supported by the Advanced Technology Center of National Astronomical Observatory of Japan and the U.S. National Science Foundation under Cooperative Agreement No PHY-0107417.

\section{References}

[1] Ando M and the TAMA Collaboration 2005 Class. Quantum Grav. 22 S881

[2] Kuroda K and the LCGT Collaboration 2006 Class. Quantum Grav. 23 S215

[3] Lück H et al 2006 Class. Quantum Grav. 23 S71

[4] Sigg A 2006 Class. Quantum Grav. 23 S51

[5] Acernese F et al 2006 Class. Quantum Grav. 23 S63

[6] Akutsu T et al 2006 Phys. Rev. D 74122002

[7] Takahashi R and the TAMA Collaboration 2004 Class. Quantum Grav. 21 S403

[8] Márka S et al 2002 Class. Quantum Grav. 191605

[9] Takamori A 2002 PhD Thesis LIGO-P-030049-00-R http://admdbsrv.ligo.caltech.edu/dcc/

[10] Takahashi R et al 2002 Rev. Sci. Instrum. 732428

[11] Takahashi R et al 2002 Class. Quantum Grav. 191599

[12] Braccini S et al 2005 Astropart. Phys. 23557

[13] Takamori A et al 2007 Nucl. Instrum. Methods A 582683 
[14] Cella G et al 2005 Nucl. Instrum. Methods A 540502

[15] Takamori A et al 2002 Class. Quantum Grav. 191615

[16] Bertolini A et al 2006 Nucl. Instrum. Methods A 556616

[17] Tariq H et al 2002 Nucl. Instrum. Methods A 489570

[18] Wang C et al 2002 Nucl. Instrum. Methods A 489563

[19] Morrison E et al 1994 Appl. Opt. 335037

[20] Morrison E et al 1994 Appl. Opt. 335041

[21] Arai K et al 2000 Phys. Lett. A 27315 